East African Medical Journal Vol. 85 No. 8 August 2008

EMERGENCY RESECTION OF SIGMOID VOLVULUS

G. Jumbi, MBChB, MMed, FCS (ECSA), DSM, Lecturer and R.T. Kuremu, MBChB, MMed, FCS (ECSA), Senior Lecturer,

Department of Surgery, Moi University School of Medicine, P.O. Box 4606, Eldoret, Kenya

Request for reprints to: Dr. G. Jumbi, Department of Surgery, Moi University School of Medicine, P.O. Box 799, Eldoret, Kenya

\title{
EMERGENCY RESECTION OF SIGMOID VOLVULUS
}

\author{
G. JUMBI and R.T. KUREMU
}

\begin{abstract}
Objectives: To determine the outcome of emergency resection of sigmoid volvulus and to determine the factors associated with adverse outcome.

Design: Retrospective case series review.

Setting: Moi Teaching and Referral Hospital (MTRH), Eldoret, Kenya.

Subjects: All cases of sigmoid volvulus operated at MTRH during the six years period between year 2000-2005.

Main outcome measures: Mortality rate; morbidity rate; and duration of hospital stay.

Results: Ninety two case files were analysed. Sigmoid volvulus accounted for $14.1 \%$ of all cases of intestinal obstruction and $80 \%$ of large gut obstruction. The mean age was 47.3 years and the median was 50 years. The range was 16 to 86 years. The male to female ratio was 29.3:1. Mortality was three $(3.3 \%)$ cases and the morbidity was $20(21.7 \%)$ cases. The mean duration of hospital stay was $\mathbf{1 1 . 8}$ days. Inadequate intravenous fluid therapy had a statistically significant adverse effect on outcome in this study. The yearly outcome remained unchanged during the six years of the study.

Conclusions: Emergency resection in cases with a viable colon had a similar outcome to the traditional standard treatment by emergency endoscopic derotation followed by semi-elective or elective resection. The overall outcome was comparable to global standards. Inadequate postoperative intravenous fluid therapy significantly affected the outcome.
\end{abstract}

\section{INTRODUCTION}

Volvulus of the sigmoid colon is the most common form of volvulus of the gastro-intestinal tract. The disease is global but it is most common in the "sigmoid belt" (Africa, parts Asia, parts of Eastern Europe and Brazil) where it accounts for up to $50 \%$ of all cases of intestinal obstruction (1). In Western Europe and North America it is responsible for up 5\% of large bowel obstruction (1). In USA, it is the third most common cause of large bowel obstruction after colorectal cancer and diverticular disease (2). There is no satisfactory explanation for the geographical distribution. It has been suggested that high fiber diet may contribute to the high incidence in Africa where the high fiber results in heavy loading of the sigmoid colon (1). In East Africa it is the second most common cause of intestinal obstruction after adhesions (3-6).

The aetiolgy is unknown. The associated factors include: confinement to mental institutions, chronic neurological disease, chronic cardiovascular disease, megacolon disease (Hirchisprungs', idiopathic, Chagas), and chronic constipation (1). Anatomical factors that favour the twisting (volvulus) are: a redundant long sigmoid loop, a long narrow sigmoid mesocolon with a narrow attachment. Other factors which favour the twisting of colon are adhesions, bands and a heavily loaded sigmoid colon.

The traditional treatment of acute volvulus of sigmoid colon with a viable colon is endoscopic derotation followed by semi-elective resection after 
three to four days or an elective resection after about four weeks. Emergency resection is traditionally done in cases with gangrenous colon only. In the last two decades, evidence based practice has led to a progressive departure from the traditional practice in favour of emergency primary anastomosis even in cases with viable colon unless there is a contraindication $(7-10,16)$. In rural setting in the developing countries, colostomy management has proved a difficult undertaking because of unacceptability of a colostomy stoma by most patients and unavailability and unaffordable cost of colostomy bags. Whenever possible, in these communities, primary anastomosis could be a more favourable option than colostomy (11). At Moi Teaching and Referral Hospital (MTRH) most cases of sigmoid volvulus are managed by emergency resection and primary anastomosis, inclusive of cases with a viable colon.

The objectives of this study were; to determine the outcome in the management of sigmoid volvulus in MTRH and to determine the factors associated with adverse outcome in the management of sigmoid volvulus in our setting.

\section{MATERIALS AND METHODS}

This study was a retrospective case series review. It was carried out in Moi Teaching and Referral Hospital in western Kenya. MTRH has 600 beds of which 100 are surgical. It is the teaching hospital for Moi University School of Medicine (MUSOM) and a referral hospital for a population of about 13 million. Cases of sigmoid volvulus are referred from as far away as $300 \mathrm{~km}$. The study material comprised of all the files for cases of sigmoid volvulus operated in Moi Teaching and Referral Hospital (MTRH) within a six year period between 1st January 2000 to 31st December 2005. All the available case files were reviewed. The exclusion criteria were: elective and semi-elective operations (four cases); case files with inadequate data (12 cases); cases files which could not be found (10 cases); and case files for deaths within 24 hours of admission (three cases). The main outcome measures were: mortality rate, morbidity rate and the average duration of hospital stay.

We collected data on the main outcome measures (mortality, morbidity, hospital stay) and data on factors that may affect the outcome. The data on morbidity (complications) included: anastomotic leakage, wound infection, wound dehiscence and post-operative fistula. Data on factors which may affect the outcome included the following: age duration of symptoms at presentation, vital signs on admission, pre-operative fluid and electrolyte imbalance (shock, sodium and potassium levels), renal function (urea and creatinine levels), pre and postoperative intravenous fluid therapy, presence of gangrene, presence of ilio-sigmoid volvulus, antibiotic treatment, type of operation (primary anastomosis or colostomy) and 'expertise' of the surgeon (whether consultant or resident/medical officer).

The data were analysed by descriptive methods using the Statistical Program for Social Sciences (SPSS-11). Correlation/association analysis was used to establish whether there was any correlation/ association between the outcome and each of the factors perceived to affect the outcome (the independent variables).

The multi-variate variables like age and outcome were dichotomised for purposes of association analysis. Depending on applicability, Fishers exact test and Chi-square test and ANOVA were used to test for statistical significance at a $p$-value $<0.05$. Logistic regression was not applicable because there was only one independent variable which showed statistically significant association with the dependent variable.

\section{RESULTS}

All patients underwent emergency resection of sigmoid colon. All received preoperative as well as postoperative antibiotics. The antibiotics included different combinations gentamycin, crystapen penicillin, amoxicillin, clavulanated amoxicillin, metronidaze, ceftiaxone and cefuroxime (depending on the available stocks in the hospital). Metronidazole featured in every combination. One hundred and sixteen cases of sigmoid volvulus were operated over the six year period of study. This was $14.1 \%$ of all cases of intestinal obstruction and $80.0 \%$ of large bowel obstruction (during this period, a total of 824 cases of intestinal obstruction and 145 cases of large bowel obstruction were treated at MTRH).

After exclusions, 92 case files were available for data analysis. There were 88 males and three females $(\mathrm{M} / \mathrm{F}$ ratio $=29.3 / 1)$. The age distribution is shown in Table 1 . The median age was 50 years (mean 
age was 47.3 years). The range was $16-86$ years. There was only one case of significant co-morbidity (renal failure) and one case reported predisposing or associated risk factors (one case of schizophrenia).

Twenty three patients (25\%) had adverse outcome (deaths and complications) while 69 patients (75\%) had no adverse outcome. Mortality was three cases $(3.3 \%)$. One patient died of multiple postoperative complications (burst abdomen, colostomy diarrhoea and feacal fistula). Another case with co-existing
$\mathrm{BPH}$ died of renal failure and the third case died of electrolyte imbalance (this patient had severely decompensated preoperative shock on admission with unrecordable BP). Morbidity was recorded in $20(21.7 \%)$ of the patients. The main causes of morbidity (complications) are shown in Table 2. There were eight cases with ilio-sigmoid knotting. Four of these had gangrene but all the eight cases had no adverse outcome.

Table 1

Age distribution of sigmoid volvulus

\begin{tabular}{lcc}
\hline Age category (years) & No. & $(\%)$ \\
\hline $10-19$ & 1 & 1.1 \\
$20-29$ & 14 & 15.2 \\
$30-39$ & 22 & 23.9 \\
$40-49$ & 14 & 15.2 \\
$50-59$ & 14 & 15.2 \\
$60-69$ & 17 & 18.5 \\
$70-79$ & 6 & 6.7 \\
$80-89$ & 2 & 2.2 \\
Not responded & 1 & 1.1 \\
\hline Total & 92 & 100 \\
\hline
\end{tabular}

Table 2

Outcome of patients with sigmoid volvulus

\begin{tabular}{lcc}
\hline & No. & $(\%)$ \\
\hline Total number of cases & 92 & 100 \\
Cases without any adverse outcome (without complications) & 69 & 75 \\
Cases with adverse outcome (deaths and complications) & 23 & 25 \\
Morbidity (Complications) & 20 & 21.7 \\
Post-operative infection & 7 & 6.6 \\
Burst abdomen & 3 & 3.3 \\
Wound dehiscence (partial). & 4 & 4.3 \\
Anastomosis leakage. & 6 & 6.5 \\
Feacal fistula & 3 & 3.3 \\
Electrolyte imbalance & 4 & 4.3 \\
Wound infection & 3 & 3.3 \\
Urinary tract infection & 2 & 2.2 \\
Chest infection & 1 & 1.1 \\
Deep vein thrombosis & 1 & 1.1 \\
Deaths (mortality) & 3 & 3.3 \\
Average duration of hospital stay & \multicolumn{2}{c}{11.8 days } \\
\hline
\end{tabular}

The Table shows the outcome (as measured by: mortality, morbidity and average duration of hospital stay). 
Table 3 shows the pattern of yearly outcome from year 2000-2005. Statistical analysis (ANOVA) of the yearly outcome showed that there was no change in mortality, morbidity and the duration of the hospital stay across the six years of the study $(\mathrm{P}=0.19)$.
We used the outcome of year 2005 as the baseline. Table 4 shows the statistical association analysis between the outcome and the factors perceived to affect the outcome. Fishers exact test or Chi-squire test (whichever was applicable) was used.

Table 3

Yearly outcome of sigmoid volvulus

\begin{tabular}{lcccccc}
\hline Year & $\begin{array}{c}\text { Total number } \\
\text { of cases }\end{array}$ & \multicolumn{2}{c}{ Mortality } & \multicolumn{2}{c}{ Morbidity } & \multicolumn{2}{c}{$\begin{array}{c}\text { Average hospital } \\
\text { stay (days) }\end{array}$} \\
\hline 2000 & $6^{*}$ & - & & - & & 14 \\
2001 & 19 & - & & 6 & 31.6 & 11.6 \\
2002 & $8^{*}$ & - & & 2 & 25.0 & 8.8 \\
2003 & 26 & 1 & 3.8 & 6 & 23.1 & 14 \\
2004 & 18 & - & - & 4 & 22.2 & 11.4 \\
2005 & 15 & 2 & 13.3 & 2 & 13.3 & 11 \\
\hline Total & 92 & 3 & 3.7 & 20 & 21.8 & 11.8 \\
\hline
\end{tabular}

${ }^{*}$ There were many cases excluded in the years 2000 and 2002 due to insufficient data

Table 4

Results of association analysis of factors affecting the outcome of sigmoid volvulus

\begin{tabular}{lccccc}
\hline Factor affecting outcome & Adverse outcome $(\mathrm{n}=23)$ & No adverse outcome $(\mathrm{n}=69)$ & \\
& No. & $(\%)$ & No. & $(\%)$ & P \\
\hline Age category & $(\mathrm{n}=23)$ & & $(\mathrm{n}=68)$ & & \\
$\quad<60$ Years & 15 & 65.2 & 51 & 75.0 & $0.3636^{1}$ \\
$\quad>60$ Years & 8 & 34.8 & 17 & 25.0 & \\
Duration of symptoms & $(\mathrm{n}=23)$ & & $(\mathrm{n}=68)$ & & \\
$\quad<3$ Days & 15 & 65.2 & 46 & 67.6 & $0.8303^{1}$ \\
$\quad>3$ Days & 5 & 27.8 & 22 & 32.4 & \\
Vital signs & $(\mathrm{n}=18)$ & & $(\mathrm{n}=54)$ & & \\
$\quad$ Abnormal & 13 & 72.2 & 44 & 81.5 & $0.4022^{1}$ \\
$\quad$ Normal & 5 & 27.8 & 10 & 18.5 & \\
Urea, electrolytes and creatinine (UEC). & $(\mathrm{n}=14)$ & & $(\mathrm{n}=47)$ & & \\
$\quad$ Abnormal & 6 & 42.9 & 30 & 63.8 & $0.6318^{1}$ \\
$\quad$ Normal & 8 & 57.1 & 17 & 36.2 & \\
Surgeon & $(\mathrm{n}=22)$ & & $(\mathrm{n}=65)$ & & \\
$\quad$ Consultant surgeon & 18 & 81.8 & 46 & 70.8 & $0.3097^{1}$ \\
$\quad$ Medical officer & 4 & 18.2 & 19 & 29.2 & \\
Type of operation & $(\mathrm{n}=22)$ & & $(\mathrm{n}=66)$ & & \\
$\quad$ Resection and primary anastomosis & 15 & 68.2 & 56 & 84.8 & $0.0864^{1}$ \\
$\quad$ Resection and colostomy & 7 & 31.8 & 10 & 15.2 & \\
Gangrene & $(\mathrm{n}=22)$ & & $(\mathrm{n}=66)$ & & \\
$\quad$ Present & 8 & 36.4 & 19 & 28.8 & $0.5046^{1}$ \\
$\quad$ Absent & 14 & 63.6 & 47 & 71.2 & \\
Pre-operative intravenous fluid (PREIVF) & $(\mathrm{n}=22)$ & & $(\mathrm{n}=43)$ & & \\
$\quad>4$ Litres in the first 24 hours & 0 & 0 & 6 & 14 & $0.3206^{2}$ \\
$\quad<4$ Litres in the first 24 hours & 12 & 100 & 37 & 86 & \\
Post-operative intravenous fluid (POSTIVF) & $(\mathrm{n}=22)$ & & $(\mathrm{n}=43)$ & & \\
$\quad>2$ Litres per day & 14 & 82.3 & 27 & 55.1 & $0.0406^{1 * *}$ \\
$\quad<2$ Litres per day & 3 & 17.7 & 22 & 44.9 & \\
\hline
\end{tabular}

${ }^{1}=$ Chi-square test ${ }^{2}=$ Fishers exact test; ${ }^{* *}=$ Significant at $\mathrm{p}=0.05$ 
Table 5

Global mortality of sigmoid volvulus

\begin{tabular}{llcc}
\hline African Series & & Number (n) & Mortality (\%) \\
\hline Sule A Z. et al 2000 (11) & Nigeria & 37 & 2.0 \\
Bhuivan M M. et al 2005 (14) & South Africa & 84 & 5.0 \\
Sule AZ. et al 1999 (15). & Nigeria & 27 & 0 \\
Jumbi G. \& Kuremu R T. 2006 (*This study) & ${ }^{*}$ Kenya & ${ }^{*} 92$ & $* 3.3$ \\
Asian series & & & \\
Kuzu MA. 2002 (16). & Turkey & 106 & 6.6 \\
Raventhiran V. 2003 (17). & India & 57 & 3.2 \\
De U. \& Ghosh S. 2003 (7). & India & 197 & 2.0 \\
Turan M. et al 2004 (18). & Turkey & 42 & 21.0 \\
Western series & & & \\
Grossman EM. et al 2000 (19). & & 178 & 14.0 \\
Salas et al 2000 (20). & USA & 49 & 8.1 \\
Connolly S. et al 2002 (21). & USA & 11 & 9.7 \\
\hline
\end{tabular}

* $=$ This study

From the above table, we observed that there was a significant association between post operative intravenous fluid therapy (POSTIVF) and the outcome ( $\mathrm{p}=0.0406)$. The odds of someone who had less than two litres/day of post operative intravenous fluid having an adverse outcome is 3.8 times that of someone who received two litres / day or more. No other independent variable in Table 4 had statistically significant association with the outcome.

\section{DISCUSSION}

The outcome of sigmoid volvulus as measured by mortality rate, morbidity rate and duration of hospital stay depends on the population studied. For example, mortality in European and Western populations is generally higher than in African populations $(12,13,19)$. All our cases were black Africans.

Some studies have reported that emergency resection has a higher mortality than semi-elective and elective resection $(12,19,29)$. All our cases were emergency resections. Table 8 compares the outcome of our series with some of other emergency resection series globally.

The above table was constructed from consecutive Pubmed literarure review for the purposes of comparison (7,14-22). Global mortalities range between $0-44 \%$ but motalities of up to $58 \%$ have been reported in Western series (13). Our yearly outcome (based on mortality, morbidity and hospital stay) remained constant between 2000-2005 (Table 3). The global mortality has also remained constant between $0-21 \%$ over the same period (see publications between 2000-2006 in Table 5). However we noted that earlier series reported higher mortality rates. For example, in 1999, Mokoena and Madiba reported a mortality of 30\% in South Africa (23) while Bak and Boley (1986), Ballantyne (1982), and Arnold F.G. and Nance G.J. (1973) reported mortalities of 36\%, 37\% and $44 \%$ respectively (13).

Our overall mortality rate of three $(3.3 \%)$ cases is among the lowest in the literature. Emergency resection has been reported to have a higher mortality than elective resection in some series $(12,19,29)$. The mortality rate in this emergency resection series is even lower than most elective and semi-elective series (12). Other measures of outcome show comparable results. Our overall morbidity rate of $20(21.7 \%)$ cases was comparable to most African and Asian series $(12,24)$ and lower than most western series.

The rate of post operative infection was $6.6 \%$ ( wound infection $=3.3 \%$, urinary tract infection $=2.2 \%$, chest infectionl=1\%) and these were in keeping with global figures. Other measures of morbidity (rates of burst abdomen (4.3\%), wound dehiscence 
$(3.3 \%)$, anastomotic leakage $(3.3 \%)$, fistula formation (3.3\%) were also comparable with results from other African and Asian series $(7,14,15,17)$. Our mean hospital stay (11.8 days) was also comparable with most series (most series reported a hospital stay of 7-14 days) $(7,14,15,21)$.

All our cases were treated by sigmoidectomy. Other types of resections (sub-total colectomy and total colectomy) were not done because we had no cases with generalised megacolon. Nonresection definitive surgical procedures (extraperitonealisation, sigmoid mesoplasty and sigmoid plaiting) are good alternatives to resection treatment bit none of our cases was treated by these procedures (31-32).

The outcome in this series of emergency resection compares very well with the traditional standard treatment (endoscopic derotation followed by semi-elective or elective resection). Kuzu reported similar results to ours in a similar emergency resection series of 106 cases (17). There are no prospective randomised case-control studies done to compare the outcome of emergency resections with elective resections in cases with a viable colon. In retrospective studies, different researchers have reported contradicting results on this issue $(15,17,29)$. One reason for these contradictions is a bias in the selection of patients. Patients undergoing emergency resections are likely to be more ill than elective cases. This bias on patient selection makes comparisons based on uncontrolled retrospective studies weak. Ideally, prospective randomised case-control studies would provide the answer but the emergency nature of the disease could make randomisation difficult or even impossible on ethical grounds. However, like in most surgical diseases, the current clinical standards in sigmoid volvulus, have been established by meta analysis of many retrospective series and not by randomised case-control studies.

We found no significant difference between cases treated by primary anastomosis (one-stage resection) and those treated by two-staged resection (colostomy) ( $\mathrm{p}=0.0864)$. However, as mentioned above, the two groups were not similar enough for a conclusive comparison. There are no prospective randomised case-control studies done to compare the outcome in primary anastomosis and twostage resections. Retrospective studies by different researchers have given conflicting results on the two types of treatment. The tread today in colonic surgery is to do more and more primary anatomosis unless there is a contra-indication $(6,17,29)$.

We investigated the effects (associations) of the following factors on the outcome: age, duration of symptoms, abnormal vital signs at admission, preoperative state of urea/creatinine/electrolytes (UEC), the type of operation procedure (primary anastomosis versus staged procedure (colostomy), adequacy of intravenous fluid therapy (both pre and postoperatively), the effect of gangrene, and the "expertise of the surgeon (consultant or resident / medical officer). In this study only postoperative fluid therapy was found to have statistically significant association with the outcome $(p=0.0406)$ (Table 4$)$. This means that our outcome could have been improved significantly by optimisation of IVF therapy. The significant association between intravenous fluid therapy and the outcome in this study underlines the importance of adequate fluid and electrolyte management in intestinal obstruction.

There was no statistically significant association between age and outcome in this study $(p=0.3636)$. In other studies, age and co-morbidity have been shown to affect the outcome adversely. The mean age in the Western study populations is 62-78 years $(19,20)$ while that of African series is almost two decades lower 42-55 years $(15,16)$.

The relatively low mortality of $3.3 \%$ in our young study population (mean age $=47.3$ years) and the relatively higher mortality rates in the older populations in the Western series suggests that advanced age has a significant bearing on the outcome. We believe that the very young study population in this series is one of the reasons for our relatively low mortality.

Co-morbidity is probably one of the most important determinant of outcome in sigmoid volvulus. Our rate of co-morbidity was remarkably low. We had only one case with benign prostatic hyperplasia with pre-existing renal failure. He was one of the three deaths. There was one case of schizophrenia which had a prolonged hospital stay (22 days). Other studies have shown that most deaths are due to co-morbidities $(7,25,29)$ particularly cardiovascular disease, chronic respiratory disease and cancer. Other co-morbidities associated with sigmoid volvulus are chronic neuro-psychiatric disease and megacolon disease. Co-morbidity rates are higher in the Western series than in the African series $(12,13)$. There is little doubt that in addition to 
the younger age, the remarkably low co-morbidity contributed markedly to our comparatively lower mortality. We found no significant difference in the outcome of patients with gangrene and those patients with a viable colon ( $p=0.5046)$.

However, the two groups of patients in these two groups were not similar enough for a conclusive comparison. Raveenthiran had similar results to ours in a similar emergency series of 57 cases (17). Other studies have reported poorer outcome among gangrenous cases. In a review article by Madiba, the average mortality for cases with a viable colon ranged between $4-12 \%$ and between $18-75 \%$ for cases with gangrene (12).

Preoperative shock at admission has been associated with poorer outcome $(8,14,16)$. We had one death out of three patients with pre-operative shock. This suggests a mortality rate of $33.3 \%$ among the cases with preoperative overt shock. We did not find any significant difference in the outcome between cases operated on by consultant surgeons and those operated on by senior resident or medical officers ( $\mathrm{p}=0.3097$ ). In many studies, Ilio-sigmoid knotting is associated with a high rate of adverse outcome $(26,27)$ but in our series, all our eight cases (four with gangrene and four without gangrene) had no adverse outcome.

In our local community, management of colostomy has proved a difficult undertaking mainly because of the negative attitude towards a colostomy stoma. Indeed, one patient threatened suicide unless the colostomy was reversed as soon as possible and three other patients refused to be discharged home until the colostomy was reversed. This negative attitude was an additional reason for doing more primary anastomoses than colostomies. Secondly, colostomy bags are not affordable and not readily available in our rural setting (15). Sule reported similar problems in management of colostomy in rural Nigeria (11).

We concur with a large body of current knowledge that if there are no high risk factors (such as advanced age or significant co-morbidity), emergency resection and primary anastomosis in acute sigmoid volvulus, is safe, cost effective, reduces the recurrence rate, reduces hospital stay and avoids a stoma (colostomy) without compromising the outcome results $(7-10,16)$.

In conclusion, the outcome of sigmoid volvulus in this study (mortality rate $=3.3 \%$, morbidity rate $=21.1 \%$ and mean hospital stay of 11.8 days) is comparable to other global outcomes. We believe that the reason for our low mortality is the absence of high risk factors such as advanced age, cardiovascular disease and neuro-psychiatric disease (low co-morbidity rate). The study corroborated results from many other studies which have shown that if there are no contraindications, emergency resection in cases with a viable colon has similar outcome to that of the traditional standard treatment by emergency endoscopic derotation followed by semi-elective (or elective) resection. In this study, inadequate post operative intravenous fluid therapy had significant association with adverse outcome. We also found out that factors commonly associated with sigmoid volvulus in the West, such as chronic neuro-psychiatric disease and chronic cardiovascular disease were prominently missing in this African series. The mean age of 47.3 years in this study is among the youngest reported. The male/female ratio of $29.3 / 1$ in this study is the highest male preponderance ratio reported. The proportion of large bowel obstruction caused by sigmoid volvulus $(80 \%)$ in this study is also the highest reported too.

We recommend emergency resection and primary anastomosis for patients without high risk factors such as advanced age and significant co-morbidity. The traditional standard method (emergency endoscopic derotation followed by semi-elective or elective resection) is recommendable for high risk patients (with a viable colon) in order to allow for maximum optimisation of the co-morbidities and any complications. Cases with gangrene must be treated by emergency resection followed by either primary anastomosis or colostomy.

Administration of intravenous fluid therapy needs to be improved at MTRH.

\section{ACKNOWLEDGEMENTS}

We wish to acknowledge the following: Director, Moi Teaching and Referral Hospital for permission of the study and the publication of this paper; Institutional Research and Ethics Committee (IREC) of MTRH and MUSOM for review and approval of the proposal; Professor J. K. Rotich for his consultancy in statistics; Ann Ndegwa for statistical analysis; Mr. Jacob Tanui for data entry; Rose Ayikukwei and Mrs. Angeline Musau and Mr. Martin Ochieng for their assistance in literature review search; All the staff of the Records Department of MTRH; and Miss. Nancy N. Kariuki for secretarial services. 


\section{REFERENCES}

1. Alan, R. Berry. Oxford Textbook of surgery. $2^{\text {nd }}$ edition 2000. Volvulus of colon. Page 1515-1519.

2. Lal, S.K., Morogenstern, R., Vinjiraver, E.F. and Martin, A. Sigmoid volvous, an update. Surgery. 1992; 112: 618-623.

3. Jani, P.G. Review of sigmoid volvulus as seen in Kenyatta National Hospital (Masters of Medicine Dissertation). June 1984. University of Nairobi.

4. Gaya, Z. Treatment of sigmoid volvulus. Proceedings of Assoc. Surgeons of East Africa. 1999; 13: 26-27.

5. Ojara, E.A. Sigmoid volvulus in Kenyatta National Hospital. East Afr. Med. J. 1983; 5:

6. Adonga, A.M. Variety of volvulus of intestine seen in Mulago Hospital, Kampala. East Afr. Med. J. 1982; 710-717.

7. De, U. and Ghosh S.A. Single stage primary anastomosis without colonic lavage for left-sided colonic obstruction due to acute sigmoid volvulus: a prospective study of one hundred and ninety-seven cases. NZ. J. Surg. 2003; 73: 390-392.

8. Dulger, M., Canturk, N.Z., Utkan, N.Z. and Gonullu N.N. Management of sigmoid colon volvulus. Hepatogastroenterology. 2000; 347: 1280-1283.

9. Trillo, C., Paris, M.F. and Brennan JT. Primary anastomosis in the treatment of acute disease of the unprepared left colon. Amer. Surg. 1998; 65: 821-825.

10. De. U. Sigmoid volvulus in rural Bengal. Trop. Doct. 2002; 32: 80-82.

11. Sule, A.Z., Obepka, P.O., Iva, D., Ogbonna, et al. Intraoperative colonic irrigation in the management of left sided large bowel emergencies in Jos University Teaching Hospital, Nigeria. East Afr. Med. J. 2000; 77: 613-617.

12. Madiba, T.E. and Thomson, S.P. The Management of sigmoid volvulus. Dis. Colon Rectum. J. R. CoIl. Surg. Edinb. 2000; 45: 125-126.

13. Anold, F.C. and Nance, G.J. Volvulus of sigmoid colon. Ann. Surg. 1973; 177: 527-537.

14. Bhuivan, M.M., Machowski, Z.A., Linyama, B.S. and Modiba, M.C. Management of sigmoid volvulus in Polokwane-Mankweng Hospital. S. Africa J. Surg. 2005; 43: 17-19.

15. Sule, A.Z., Iva, D., Obekpa, P.O., Ogbonna, B., et al. Onestage procedure in the management of acute sigmoid volvulus. J. R. Coll. Surg. Edinb. 1999; 44: 164-166.

16. Kuzu, M.A., Aslar, A.K., Soran, A., et al. Emergent resection for acute sigmoid volvulus: results of 106 consecutive cases. Dis. Colon Rectum. 2002; 45: 1085-1090.
17. Raveenthiram, V. Restorative resection of unprepared left gangrenous vs viable sigmoid volvulus. Int. J. Colorectal Dis. 2004; 19: 58-60.

18. Turan, M.O., Sen, M., Karadavi, K., Koyuncu, A., Topcu, O., Yildirir, C. and Duman, M. Rev. Esp. Enferm. Dig. 2004; 96: 32-35.

19. Grossmann, E.M., Longo, W.E., Stratton, M.D., et al. Sigmoid volvulus in Department of Veterans Affairs Medical Centres. Dis. Colon Rectum. 2000; 43: 414-418.

20. Salas, S., Angel, C.A., Salas, N., Murillo, C. et al. Sigmoid volvulus in children and adolescents. J. Amer. Coll. Surg. 2000; 190: 717-723.

21. Connolly, S., Brannigan, A.E., Heffeman, E., et al. Sigmoid volvulus: a 10-year-audit. Ir. J. Med. Sci. 2002; 171: 216-217.

22. Patriti, A., Contine, A., Carbone, E., Gulla, N. and Donini, A. One-stage resection without colonic lavage in emergency surgery of the left colon. Colorectal Dis. 2005; 7: 332.

23. Mokoena, T.R. and Madiba, T.F. Sigmoid volvulus among Africans in Durban. Trop. Geogr. Med. 1995; 47: 216-217.

24. Renzulli, P., Maurer, C.A., Netzer, P. and Buchler, M.W. Preoperative colonoscopic derotation is beneficial in acute colonic volvulus. Dig. Surg. 2002; 19: 223-

25. Hiltunen, K.M., Syria, H. and Matikainen, M. Colonic volvulus. Diagnosis and results treatment in 82 patients. Eur J Surg. 1992; 158: 607-611.

26. Mallick, I.H. and Winslet, M.C. Ileosigmoid knotting. Colorectal Dis. 2004; 6: 220-225.

27. Gibney, E.J. and Mock, O.N. Ileosigmoid knotting. Dis. Colon Rectum. 1993; 36: 855-857.

28. Agaoglu, N., Yucel, Y. and Turkyilmaz, S. Surgical treatment of the sigmoid volvulus. Acta Chir Belg. 2005; 105: 365-368.

29. Hamed, I.A. Recurrent colonic volvulus in children. Paediatr. Surg. 1997; 32: 1739-1742.

30. Bhatnagar, B.N. and Sharma, C.L. Nonresective for the cure of non-gangrenous sigmoid volvuls. Dis. Colon Rectum. 1998; 41: 381-388.

31. Bach, O., Rudloff, U. and Post, S. Modification of mesosigmoidoplasty for nongagrenous sigmoid volvulus. World J. Surg. 2003; 27: 1329-1332.

32. Altarac, S., Glavas, M., Drazinic, K., Kovac, D. et al. Experimental and clinical study in the treatment of sigmoid volvulus. Acta. Med. Croaticu. 2001; 55: 67. 